\title{
The diagnostic value of normalized ADC using spleen as reference organ in assessment liver fibrosis
}

\author{
Mai A. Mostafa ${ }^{1}$, Omnia Kamal ${ }^{1}$, Aya Yassin ${ }^{1 *}$ D, Mona A. Nagi ${ }^{1}$, Osama A. Ahmed ${ }^{2}$ and Hagar A. Ahmed ${ }^{2}$
}

\begin{abstract}
Background: To investigate the value of liver ADC normalization using spleen as a reference organ in liver fibrosis assessment compared to Fibroscan.

A total of 60 participants were included, 30 HCV positive patients and 30 in control group. We calculated mean spleen apparent diffusion coefficient (ADC), liver mean ADC, and normalized liver ADC (defined as the ratio of liver $A D C$ to spleen $A D C$ ) which were compared between cirrhotic patients and the control group. Data was analyzed, and ROC was used to evaluate the performance of nADC.

Results: No significant difference between spleen ADC values of patient and control groups or in-between different fibrosis stages. A negative correlation between liver ADC and $\mathrm{nADC}$ values with increasing fibrosis stages. We also found that the mean liver ADC and $\mathrm{ADC}$ value in patients with hepatic fibrosis were significantly lower than that of control group $\left(1.53 \times 10^{-3} \mathrm{~mm}^{2} / \mathrm{s}\right.$ vs $\left.1.65 \times 10^{-3} \mathrm{~mm}^{2} / \mathrm{s}\right)$. After analysis with $\mathrm{ROC}$, nADC shows higher diagnostic performance compared to liver ADC. nADC area under the curve (AUC) was 0.878 for detection of stage $\geq F 2$ with sensitivity and specificity of $87 \%$ and $80 \%$ respectively while ADC AUC was 0.548 with sensitivity and specificity of $62 \%$ and $72 \%$ respectively $(p=0.021) ; \geq F 3$ AUC of nADC was 0.891 with sensitivity and specificity of $88.7 \%$ and $80 \%$ respectively while ADC AUC is 0.603 with sensitivity and specificity of $72 \%$ and $72 \%$ respectively $(p=0.023)$, and F4 stage nADC AUC was 0.879 for with sensitivity and specificity of $90 \%$ and $80 \%$ respectively, while ADC AUC was 0.648 with sensitivity and specificity of $80 \%$ and $72 \%$ respectively $(p=0.054)$.
\end{abstract}

Conclusion: Normalized liver ADC using the spleen as reference organs increases the diagnostic performance of $M R$ in evaluation liver fibrosis compared to ADC alone.

Keywords: MRI DWI, Hepatitis C virus, Liver fibrosis, Liver cirrhosis, Fibroscan

\section{Background}

HCV (hepatitis $C$ virus) causes liver cell injury which may be acute or chronic. In the chronic stage, the injury by fibrous tissue accumulates in extracellular space. Hepatitis $C$ viral infection is the most common cause of liver fibrosis in Egypt [1].

\footnotetext{
* Correspondence: ayassin@hotmail.com

${ }^{1}$ Radiology Department, Faculty of Medicine, Ain Shams University, Cairo, Egypt

Full list of author information is available at the end of the article
}

Liver fibrosis plays a rule in other liver lesions pathogenesis as HCC (hepatocellular carcinoma), portal hypertension, and cirrhosis [2].

The assessment of liver fibrosis in patients with viral hepatitis is essential to follow-up on the disease progression as well as to detect patients who need antiviral treatment [3].

Liver biopsy is the gold standard for evaluating changes in fibrosis. It is considered a safe procedure but still invasive and had some contradictions and complications [4]. So, we needed a non-invasive procedure that offers a safe, fast, and reproducible way for assessing hepatic fibrosis to monitor the response of treatment [5].
Springer Open (c) The Author(s). 2020 Open Access This article is licensed under a Creative Commons Attribution 4.0 International License, which permits use, sharing, adaptation, distribution and reproduction in any medium or format, as long as you give appropriate credit to the original author(s) and the source, provide a link to the Creative Commons licence, and indicate if changes were made. The images or other third party material in this article are included in the article's Creative Commons licence, unless indicated otherwise in a credit line to the material. If material is not included in the article's Creative Commons licence and your intended use is not permitted by statutory regulation or exceeds the permitted use, you will need to obtain permission directly from the copyright holder. To view a copy of this licence, visit http://creativecommons.org/licenses/by/4.0/. 
Non-invasive radiological methods that were investigated are Fibroscan, (MRE) magnetic resonance elastography, diffusion-weighted imaging (DWI), perfusionweighted imaging, and MR spectroscopy [6].

Fibroscan is a non-invasive method for evaluating liver fibrosis in chronic liver injury. However, it has some limitations in different situations as in obese patients, ascites, and narrow intercostal spaces [7].

Diffusion-weighted imaging (DWI) is also a promising non-invasive method as it assesses tissue diffusivity which is being low in $\mathrm{HCV}$ patients with fibrosis due to the accumulation of fibrous tissue within the extracellular matrix. Multiple studies confirmed this hypothesis as patients with livers fibrosis had low ADC compared to normal individuals [8].

However, other studies revealed using different $b$ values could lead to variable ADC measurements as well as breathing methods like breath-hold, free-breathing, or respiratory triggered techniques, which can affect ADC quantification [9].

To reduce the variability of $\mathrm{ADC}$ values, researchers suggested that normalization of ADC using a reference organ that remains relatively constant across patients or systems could be the solution to this problem. Multiple studies showed that normalized ADC (using the spleen) as reference organ improves the diagnostic performance in assessing liver fibrosis than using ADC alone. The spleen may be an ideal reference organ because it maintains a relatively stable ADC even in the setting of liver disease [10].

\section{Methods}

\section{Subjects}

A total of $30 \mathrm{HCV}$ patients were included in the study after obtaining approval from the local ethics committee and informed consent from the patients. The clinical diagnosis of all the patients was established based on the patient's clinical symptoms, physical findings, and laboratory tests. The ADC values of the patients were compared with their normal counterparts. The control group for this study consisted of another 30 patients who underwent abdominal MRI owing to pancreatic, adrenal, and renal pathology but who had no known hepatic disorders.

\section{Technique of Fibroscan}

All patients were examined by TE, transient elastography (Fibroscan ${ }^{\circ}$ 502; Echosens, Paris, France). Measurements of liver stiffness were performed by 5 years experienced internist on the right lobe of the liver through intercostal spaces in correspondence to the mid-axillary line, while patients are lying in the supine position with the right arm in maximal abduction. A median of 12 valid readings was calculated. The results were expressed in kilopascals $(\mathrm{kPa})$ and interpreted as follows: F0 (control group), < $5 \mathrm{kPa}$; F1, 5-7 kPa; F2, 7-9.5 kPa; F3, 9.5-12.5 $\mathrm{kPa}$; and F4, > $12.5 \mathrm{kPa}$.

\section{MRI examination}

1.5T machine (Achieva, Philips medical system, Eindhoven, Netherlands), using Torso phased-array coil to acquire DWI of liver and spleen, three $b$ values were taken of $0,400,800 \mathrm{~mm}^{2} / \mathrm{s}$, then on FujifiLN, synapse 3D, and V4.4EU. We calculated mean spleen apparent diffusion coefficient (ADC), liver mean ADC, and normalized liver $\mathrm{ADC}$ (defined as the ratio of liver ADC to spleen ADC) which were compared between cirrhotic patients and patients in the control group

\section{The examination protocol included}

DWI performed using single-shot echo-planar imaging (EPI) fat suppressed sequence in the axial plane, during a single end-expiratory breath-hold, using $b$ values of 0 , 400 , and $800 \mathrm{~s} / \mathrm{mm}^{2}$ using the following parameters (Table 1).

\section{Quantitative image analysis}

A single radiologist with 3 years of experience in body MR imaging drew regions of interest (ROIs) on DWI, and they were identically positioned on corresponding ADC maps by applying the copy and paste functions of the PACS system at the same points measured by the Fibroscan. The surface area of the ROIs was $20 \mathrm{~mm}^{2}$. Five ROIs inserted on the liver one at the left lobe, one at the caudate lobe, and 3 separate points in the right lobe as follows: one at the anterior segment and two at the posterior segment, one at segment 6 , and other at segment 7 . The mean hepatic ADC value was calculated by taking the mean of the total of five ADC measurements obtained. For the spleen ADC measurements, a circular ROI of approximately $20 \mathrm{~mm}^{2}$ was used. The measurements were taken from parenchyma areas at

Table 1 DWI performed using single-shot echo-planar imaging (EPI) fat suppressed sequence in the axial plane, during a single end-expiratory breath-hold, using b-values of 0,400 , and $800 \mathrm{~s} /$ $\mathrm{mm} 2$ using the following parameters

\begin{tabular}{ll}
\hline & DWl \\
\hline TR (ms) & $1,600-3,400$ \\
TE $(\mathrm{ms})$ & 80 \\
SNR & 0 \\
NEX & 2 \\
ST (mm) & 3 \\
Gap $(\mathrm{mm})$ & 0 \\
FOV $(\mathrm{cm})$ & 421 \\
Matrix & Up to $256 \times 256$ \\
\hline
\end{tabular}


least $1 \mathrm{~cm}$ away from the capsule and not crossing major vascular structures as far as possible. Three ROIs were drawn as one each from the inferior pole, the interpolar section, and the superior pole. The mean spleen ADC value was calculated from the mean of the three measurements (Fig. 2). Then, normalized ADC value was calculated by dividing mean liver ADC over mean spleen ADC.

\section{Data management and analysis}

The collected data was revised, coded, tabulated, and introduced to a PC using Statistical package for Social Science (SPSS 20). Data was presented, and suitable analysis was done according to the type of data obtained for each parameter.

\section{Analytical statistics}

One tailed $t$-test is to compare between control and patients' groups.

Mann-Whitney test ( $U$ test) was used to assess the statistical significance of the difference of a nonparametric variable between two study groups.

Correlation analysis (using Spearman's method) is to assess the strength of association between two quantitative variables. The correlation coefficient denoted symbolically " $r_{s} r$ " defines the strength (magnitude) and direction (positive or negative) of the linear relationship between two variables. Liver ADCs, spleen ADCs, and normalized liver ADCs were correlated with fibrosis scores.

The ROC curve (receiver operating characteristic) provides a useful way to evaluate the sensitivity and specificity for quantitative diagnostic measures that categorize cases into one of two groups.

Differences in ADC values were statistically significant when $p<0.05$.

\section{Results}

Total participants are 60 in this study: 30 controls (19 females and 11 males of mean age $34 \pm 10$ years) and 30 patients: 16 females and 14 males with mean age $47 \pm$ 11 , F1 $(n=6)$, F2 $(n=6)$, F3 $(n=8)$, and F4 $(n=10)$.
There was significant difference between the mean liver $\mathrm{ADC}$ value of the liver in the control group $(1.65 \times$ $\left.10^{-3} \mathrm{~mm}^{2} / \mathrm{s}\right)$ compared to patients $\left(1.53 \times 10^{-3} \mathrm{~mm}^{2} / \mathrm{s}\right.$ with $p=0.04)$. As well as the nADC, there was significant difference between cases $(\mathrm{nADC}=1.39)$ and controls (nADC $=1.87$ with $p=0.006)$ (Table 2).

\section{Correlations between fibrosis stage, liver ADC, and normalized liver ADC}

- Mean ADC of the liver and nADC value show negative correlation with increasing fibrosis stages (Table 3 and Fig. 1), but there were some overlaps in different stages of fibrosis.

\section{Correlations between fibrosis stage and spleen ADC}

There was no significant difference between mean spleen ADC values among participants with different fibrosis stages (Fig. 2).

\section{Correlations between liver $A D C$, normalized $A D C$, and spleen ADC}

- There was no significant correlation between mean liver $\mathrm{ADC}$ or $\mathrm{nADC}$ and mean spleen $\mathrm{ADC}$ in the 30 cases (Fig. 3).

\section{Differentiating fibrosis stages using $A D C$ and $\mathrm{nADC}$}

- Mean liver ADC could not differentiate each individual stages of fibrosis except between F0 versus F4.

- nADC has significant differences between F0 and F2, F3, and F4 (Table 4).

\section{Diagnostic performance of $A D C$ and $n A D C$ using of} receiver operating characteristic (ROC) curve analysis for each stage

According to ROC analysis, nADC exhibited a good diagnostic performance for each stage of fibrosis, with AUCs higher than those of ADC.

The optimal cut-off values of nADC liver and ADC for each fibrosis stages are shown in Table 5.

Table 2 Mean liver and spleen ADC and nADC in control and patients' groups

\begin{tabular}{|c|c|c|c|c|c|c|}
\hline & & Control group $(\mathrm{No}=30)$ & Patient group $(\mathrm{No}=30)$ & t-test & $P$-value & Sig \\
\hline \multirow[t]{2}{*}{ Mean ADC liver } & Mean $\pm S D$ & $1.65 \pm 0.9$ & $1.53 \pm 0.41$ & 1.72778 & $0.0468^{*}$ & $S$ \\
\hline & Range & $0.9-2.3$ & $0.9-2$ & & & \\
\hline \multirow[t]{2}{*}{ Mean ADC spleen } & Mean $\pm S D$ & $0.85 \pm 1.61$ & $1 \pm 0.33$ & 1.29422 & 0.242 & NS \\
\hline & Range & $0.6-1.8$ & $0.8-1.7$ & & & \\
\hline \multirow[t]{2}{*}{ Normalized ADC } & Mean \pm SD & $1.87 \pm 0.50$ & $1.39 \pm 0.49$ & -6.12057 & $0.001^{*}$ & S \\
\hline & Range & $1.1-3$ & $0.94-2$ & & & \\
\hline
\end{tabular}


Table 3 Negative correlation was noted between liver ADC value as well as nADC with increasing fibrosis stage

\begin{tabular}{lllll}
\hline Fibrosis stage & NO & Mean Liver ADC $\left(\times 10^{-3} \mathrm{~mm}^{2} / \mathrm{s}\right)$ & $\begin{array}{l}\text { Mean Spleen } \\
\text { ADC }\left(\times 10^{-3} \mathrm{~mm}^{2} / \mathrm{s}\right)\end{array}$ \\
\hline Control group F0 & 30 & 1.65 & 0.87 & 1.8 \\
F1 & 6 & 1.59 & 1.1 & 1.45 \\
F2 & 6 & 1.55 & 1.13 & 1.42 \\
F3 & 8 & 1.5 & 1 & 1.4 \\
F4 & 10 & 1.48 & 1 & 1.38 \\
\hline
\end{tabular}

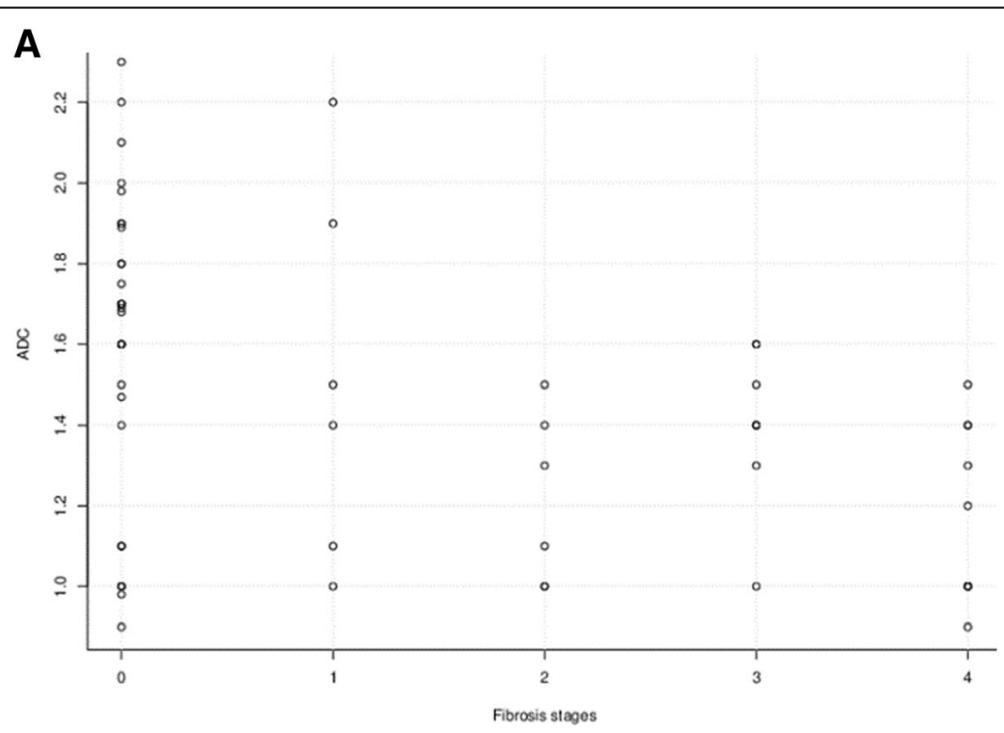

B

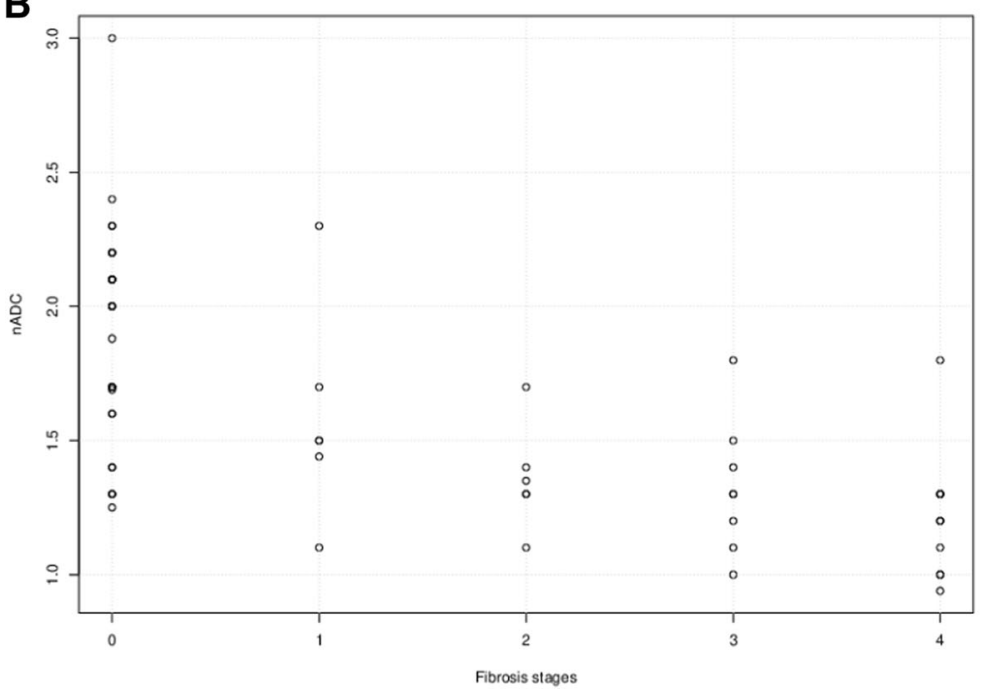

Fig. 1 a, b shows negative correlation between mean liver ADC of control group and different fibrosis stages groups. (Spearman's rank correlation coefficient $r s=-0.6,95 \% \mathrm{Cl}:-0.446-0.015, p=0.0374)$, also negative correlation between nADC of control group and different liver fibrosis stages groups. (Spearman's rank correlation coefficient $r s=-0.82,95 \%$ Cl:- $0.634-0.285, p<0.001$ ) 


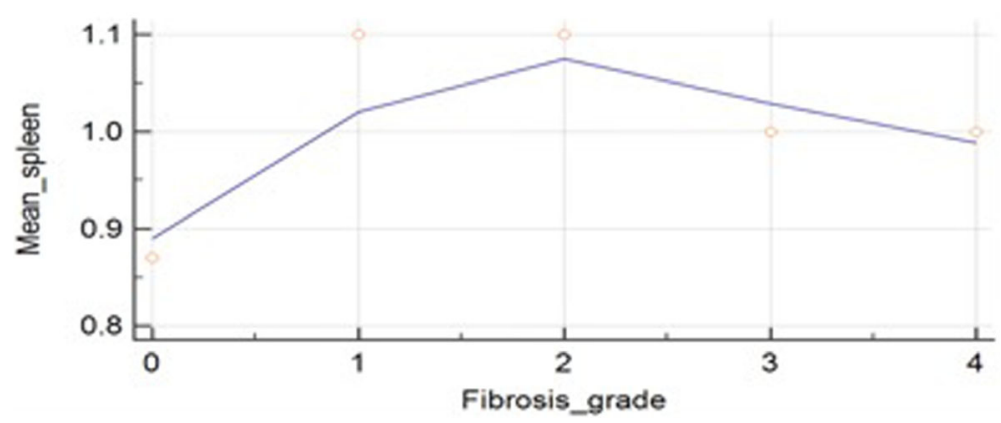

Fig. 2 Shows no significant correlation between mean spleen ADC in different fibrosis stages. (Spearman's rank correlation coefficient $r s=0.105$; $95 \% \mathrm{Cl}:-0.153-0.350, p=0.866)$.

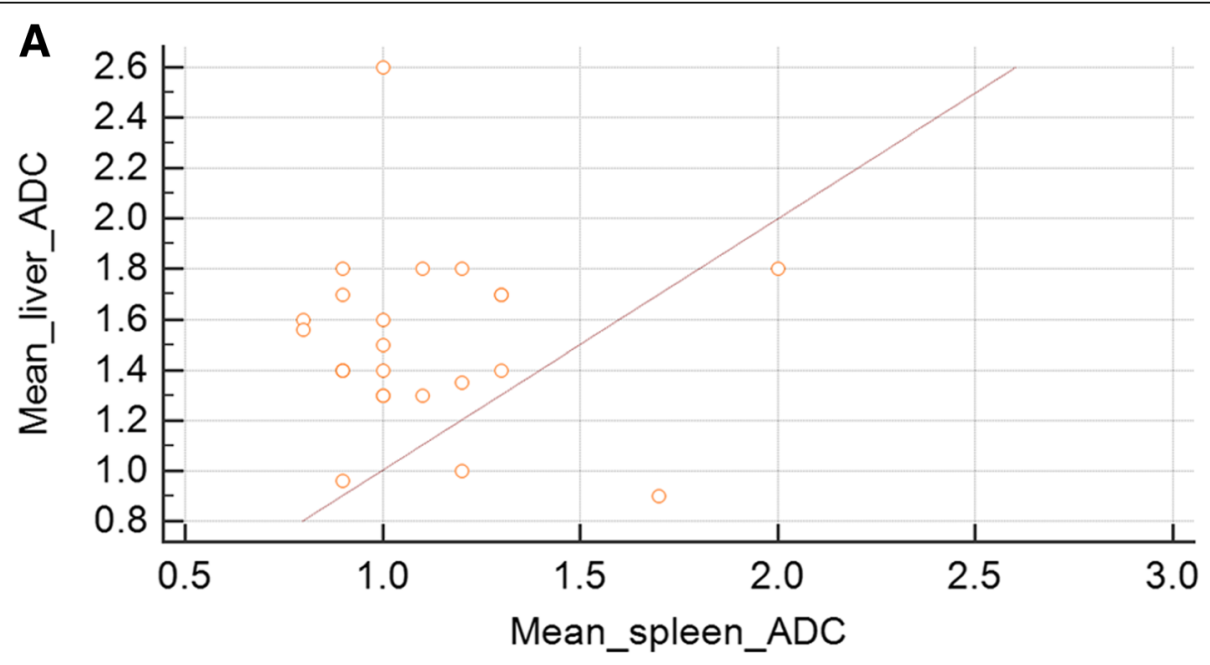

B

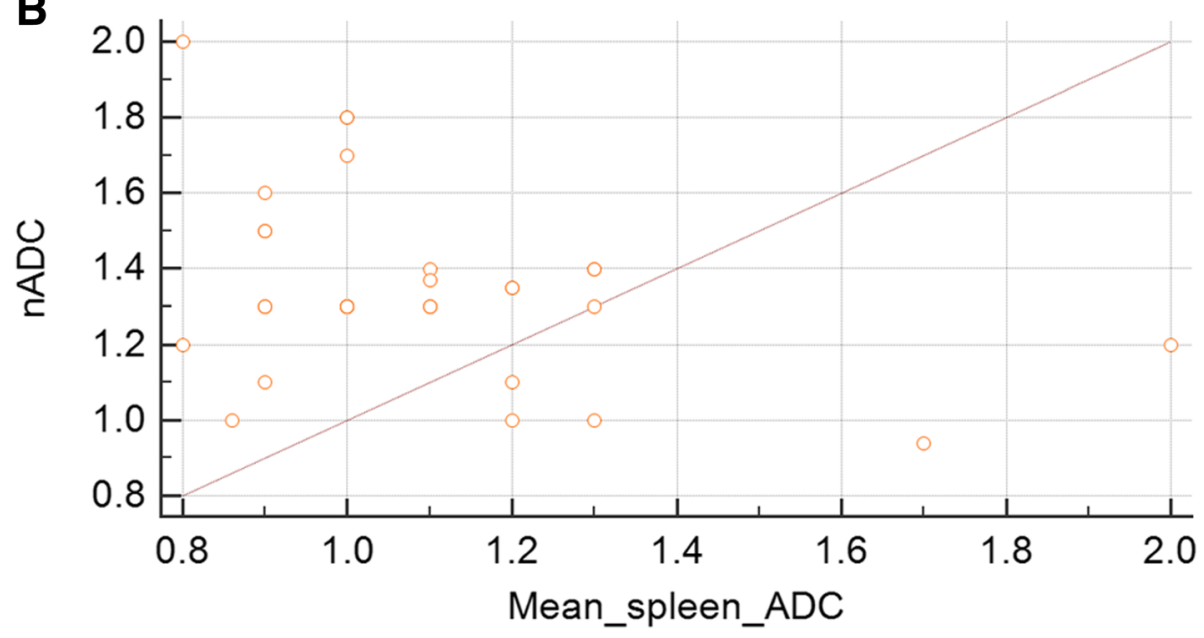

Fig. $3 \mathbf{a}$, $\mathbf{b}$ shows no significant correlation between liver and mean spleen ADC $(n=30)$ of patients with different stages of fibrosis (Spearman's rank correlation coefficient $r s=0.00335 ; 95 \% \mathrm{Cl}:-0.357-0.363, p=0.9$ ), also no significant correlation between nADC and mean spleen ADC(Spearman's rank correlation coefficient $r s=-0.225 ; 95 \% \mathrm{Cl}:-0.538-0.125, p=0.1743)$. (Image quality adjusted) 
Table 4 ADC and nADC comparison among different fibrosis stages

\begin{tabular}{lll}
\hline Fibrosis stage & Liver ADC $p$-value & nADC $p$-value \\
\hline F0 vs F1 & 0.63836 & 0.05574 \\
F0 vs F2 & 0.5485 & $0.01287^{*}$ \\
F0 vs F3 & 0.13888 & $0.00138^{*}$ \\
F0 vs F4 & $0.02869^{*}$ & $0.00026^{*}$ \\
\hline
\end{tabular}

*=significant $p$-value using Mann-Whitney U Test

\section{Comparing diagnostic performance of liver apparent diffusion coefficient (ADC) versus normalized liver ADC for detection of fibrosis stages $\geq F 2, \geq F 3$, and F4 using ROC curves analysis}

According to ROC curves analysis, nADC liver exhibited a good diagnostic performance for moderate (> F2) and severe (> F3) fibrosis stages compared to ADC as they show a larger area under the curve (AUCs) (Table 6, Fig. 4). There is a statistically significant difference between the area under the receiver operating characteristic curve (AUC) of nADC liver vs ADC liver except for the F4 stage; our results were as follows (Figs. 5, 6, 7):

Table 5 The optimal cut-off values of nADC liver and ADC for each fibrosis stages

\begin{tabular}{lll}
\hline Variable & ADC & nADC liver \\
\hline F0 vs F1 & & \\
Optimal cut-off value & $1.55\left(\times 10^{-3} \mathrm{~mm}^{2} / \mathrm{s}\right)$ & 1.44 \\
Sensitivity (\%) & $70 \%$ & $83 \%$ \\
Specificity (\%) & $66 \%$ & $67 \%$ \\
AUC (95\% Cl) & $0.588(379$ to 775$)$ & $0.717(507$ to 874$)$ \\
F0 vs F2 & & \\
Optimal cut-off value & $1.53\left(\times 10^{-3} \mathrm{~mm}^{2} / \mathrm{s}\right)$ & 1.41 \\
Sensitivity (\%) & $72 \%$ & $84 \%$ \\
Specificity (\%) & $55 \%$ & $66 \%$ \\
AUC (95\% Cl) & $0.617(407$ to 799$)$ & $0.729(520-883)$ \\
F0 vs F3 & & \\
Optimal cut-off value & $1.51\left(\times 10^{-3} \mathrm{~mm}^{2} / \mathrm{s}\right)$ & 1.4 \\
Sensitivity (\%) & $80 \%$ & $80 \%$ \\
Specificity (\%) & $50 \%$ & $75 \%$ \\
AUC (95\% Cl) & $0.644(442-818)$ & $0.739(565-902)$ \\
F0 vs F4 & & 1.38 \\
Optimal cut-off value & $1.49\left(\times 10^{-3} \mathrm{~mm}^{2} / \mathrm{s}\right)$ & $90 \%$ \\
Sensitivity (\%) & $80 \%$ & $80 \%$ \\
Specificity (\%) & $72 \%$ & $0.879(589-908)$ \\
AUC (95\% Cl) & $0.648(453-812)$ & \\
\hline
\end{tabular}

1. Fibrosis stage $\geq \mathrm{F} 2,0.878$ vs $0.548(p=0.021)$;

2. Fibrosis stage $\geq$ F3, 0.891 vs $0.603(p=0.023)$; and

3. Fibrosis stage F4, 0.879 vs $0.648(p=0.054)$.

In our study, normalized ADC was done on a single patient with moderate ascites, but he was excluded as Fibroscan results are not accurate in cases with ascites. It was classified as F4 by Fibroscan while mean liver $\mathrm{ADC}$ value $=1.5$ and mean spleen $\mathrm{ADC}=1.07$ and $\mathrm{nADC}=1.4$ (Fig. 8).

\section{Discussion}

Hepatitis $\mathrm{C}$ virus (HCV) is the main agent of posttransfusion chronic hepatitis and liver fibrosi $\mathrm{s}$ [11]. The highest incidence of HCV infection in the world is in Egypt [12], which makes a need for different laboratory and radiological methods to asses liver injury [13].

In the last few years, Fibroscan was considered as a fast, safe, and reproducible procedure to assess liver stiffness to predict liver fibrosis stag e[14-16].

However, Fibroscan cannot be used in obese patients, ascites, and narrow intercostal spaces [17].

Diffusion-weighted imaging enables qualitative and quantitative assessment of tissue diffusivity. Random motion of water molecules in the liver can be quantified by calculation of the apparent diffusion coefficient (ADC). The ADC of livers with moderate or advanced fibrosis and cirrhosis has been reported to be lower than that of normal livers or livers with mild fibrosis across multiple studies [18].

DWI is a crucial sequence in the MR abdomen, but the lack of standardization of DWI acquisition techniques is a major limitation of more broad and uniform use of ADC measurements as a quantitative biomarker. The variability in reported ADC values is further complicated by the use of different $b$ values and acquisition methods based on breath-hold, freebreathing, or respiratory triggered techniques, which can affect ADC quantification and that should have influenced all of the abdominal organs scanned, including not only the liver, but also the spleen, pancreas, kidney, and paraspinal muscle. So, in our study as well as previous studies, we assume that normalization using a reference organ would not be affected by different respiratory motion compensation techniques [19].

Many researchers have tried to evaluate the impact of the apparent diffusion coefficient (ADC) measurements in the assessment of liver diseases. Many studies show that the cirrhotic livers are of lower ADC values than the healthy livers [20]. This is consistent with our study as we also demonstrated a linear negative correlation between liver ADC value and fibrosis stages $(r=-0.63)$. 
Table 6 nADC liver exhibited good diagnostic performance for each stage of fibrosis Compared to ADC liver

\begin{tabular}{|c|c|c|c|c|c|c|c|}
\hline \multicolumn{4}{|c|}{ Liver ADC } & \multicolumn{4}{|l|}{$\mathrm{nADC}$} \\
\hline Stage & $A \cup C$ & Sensitivity & Specificity & AUC & Sensitivity & Specificity & $p$-value \\
\hline$\geq 2$ & 0.548 & $62 \%$ & $72 \%$ & 0.878 & $87 \%$ & $80 \%$ & 0.021 \\
\hline $3-4$ & 0.603 & $72 \%$ & $72 \%$ & 0.891 & $88.8 \%$ & $80 \%$ & 0.023 \\
\hline 4 & 0.648 & $80 \%$ & $72.2 \%$ & 0.879 & $90 \%$ & $80 \%$ & 0.054 \\
\hline
\end{tabular}

We also found that the mean liver ADC value in patients with hepatic fibrosis was significantly lower than that of volunteers $\left(1.47 \times 10^{-3} \mathrm{~mm}^{2} / \mathrm{s}\right.$ vs $1.65 \times 10^{-3} \mathrm{~mm}^{2} / \mathrm{s}$, $p=0.04)[21,22]$.

In another study done by Sandrasegaran et al. [23], ADC was able to differentiate cirrhotic from the noncirrhotic liver with lower ADC values of the former; however, in that study, ADC could not categorize liver fibrosis in different stages; this is also confirmed by Razek et al. [24]. Earlier study done by Do et al. [25] concluded that liver ADC failed to distinguish individual stages of fibrosis, except between stages 0 and $4 \mathrm{~mm}$; these results are the same to ours.

The variability in reported ADC values is further complicated using different $b$ values and acquisition methods based on breath-hold, free-breathing, or respiratory triggered techniques, which can affect ADC quantification $[26,27]$. For example, in our study, mean liver ADC $(b=$ 800 ) for the control group was $1.65 \pm 0.44 \times 10^{-3} \mathrm{~mm}^{2} / \mathrm{s}$ and $\mathrm{nADC}$ liver $1.87 \pm 0.50$, while in a recent study done by Shin et al. [28], the mean liver ADCs for the control group (F0) was $1.389 \times 10^{-3} \mathrm{~mm}^{2} / \mathrm{s}$, and nADC liver was 1.977.

In the study carried out by Kim et al. [29], there was no difference between spleen ADCs values between diseased and volunteers' individuals. This supports our results as there was no significant difference between spleen ADC values among patients in comparison to control $\left(1 \pm 0.33 \times 10^{-3} \mathrm{~mm}^{2} / \mathrm{s}\right.$ vs $0.85 \pm 1.61 \times 10^{-3}$ $\left.\mathrm{mm}^{2} / \mathrm{s}, p=0.24\right)$; also, there was no significant difference in the spleen ADC between different stages of fibrosis $(r=0.105 ; p=0.866)$.

Pervious results of Do et al. [25] showed that area under ROC curve values of $\mathrm{nADC}$ were higher than liver ADC values. AUC of $\mathrm{nADC} \geq \mathrm{F} 2$ stages was 0.864 with sensitivity and specificity of $90 \%$ and $77 \%$ respectively while ADC values were 0.655 with sensitivity and specificity of $61 \%$ and $61 \%$ respectively ( $p=0.013)$; AUC of $\mathrm{nADC} \geq \mathrm{F} 3$ stages was 0.805 with sensitivity and specificity of $96 \%$ and $71 \%$ respectively while ADC values were 0.689 with sensitivity and specificity of $56 \%$ and $71 \%$ respectively $(p=0.015)$, and nADC AUC for F4 stage was 0.935 with sensitivity and specificity of $95 \%$ and $66 \%$ respectively while ADC AUC was 0.720 with sensitivity and specificity of $76 \%$ and $60 \%$ respectively $(p=0.185)$.

While our study reveals close results, there was a statistically significant difference between area under the receiver operating characteristic curve (AUC) of
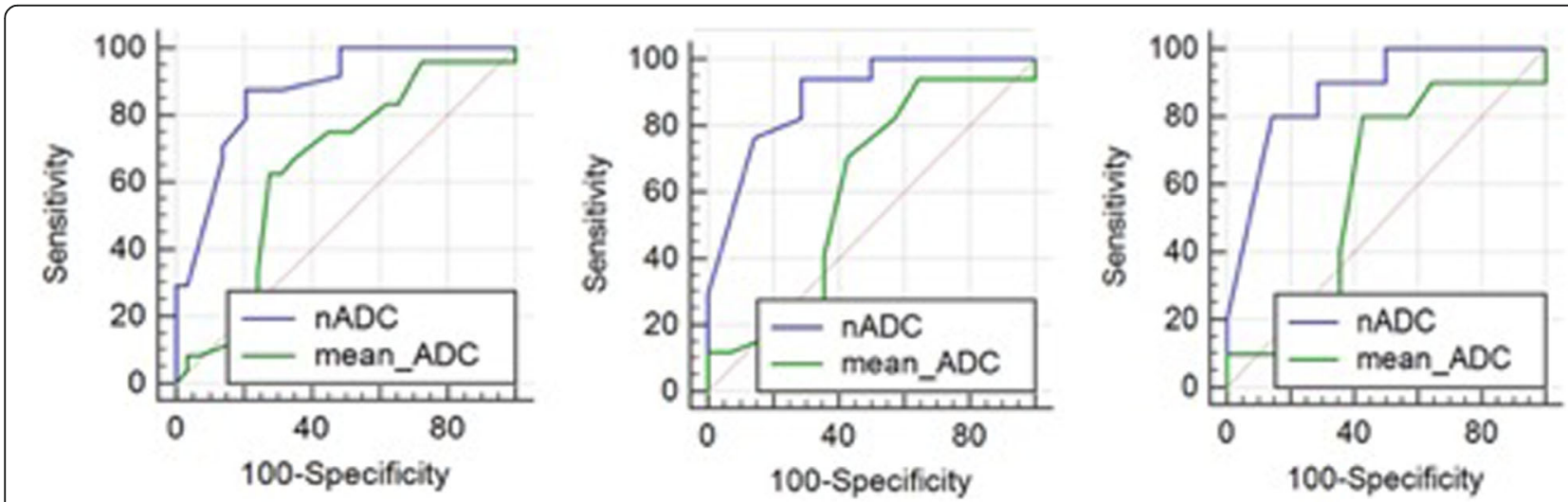

Fig. 4 Receiver operating characteristic curves (ROC) for liver apparent diffusion coefficient (ADC; green line) and normalized liver ADC (blue line). It differentiates the patients into different fibrosis stages (A-detection of stage $\geq 2$, left curve; B-detection of stage $\geq 3$, middle curve; C-detection of cirrhosis [stage 4], right curve) 


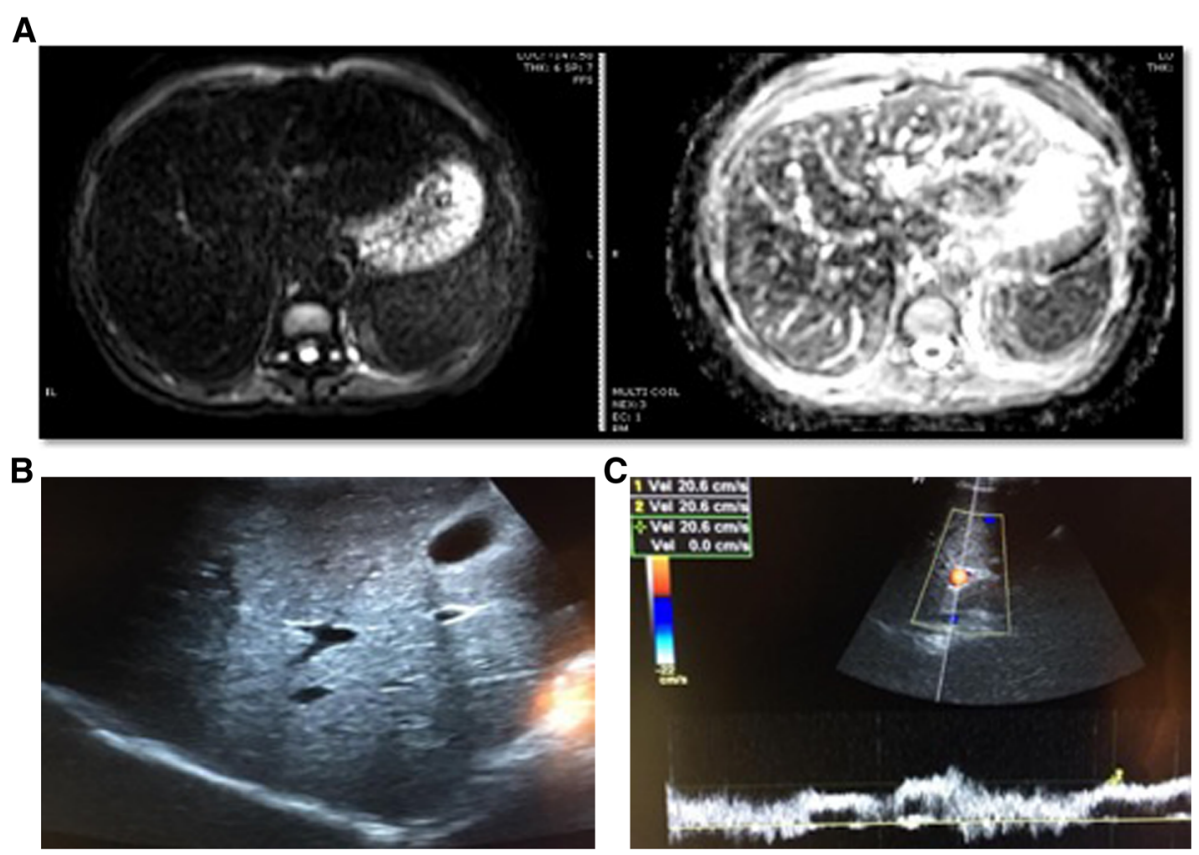

Fig. 5 a 35 years old HCV positive female patient, underwent Fibroscan with $6 \mathrm{kPa}$ which was classified as F1 stage ( $\mathrm{F} 1=5-7 \mathrm{kPa})$, His diffusion study: reveals mildly enlarged liver with no focal lesions. The spleen was of average size with no focal lesions (a)-DWl at $b=800-$ and (b)-ADC map, revealed mean liver $A D C=0.95 \times 10-3 \mathrm{~mm} 2 / \mathrm{s}$ and spleen mean $A D C=0.66 \times 10-3 \mathrm{~mm} 2 / \mathrm{s}$ so normalized $A D C=1.45(F 1 \mathrm{nADC}$ cut off $=$ 1.44). b \& c -His ultrasound study reveals (b)-mild hepatomegaly $(16 \mathrm{~cm})$ with no cirrhotic features or focal lesions and (c)-portal vein shows average calibre with the hepatopedal flow( numbering adjusted)
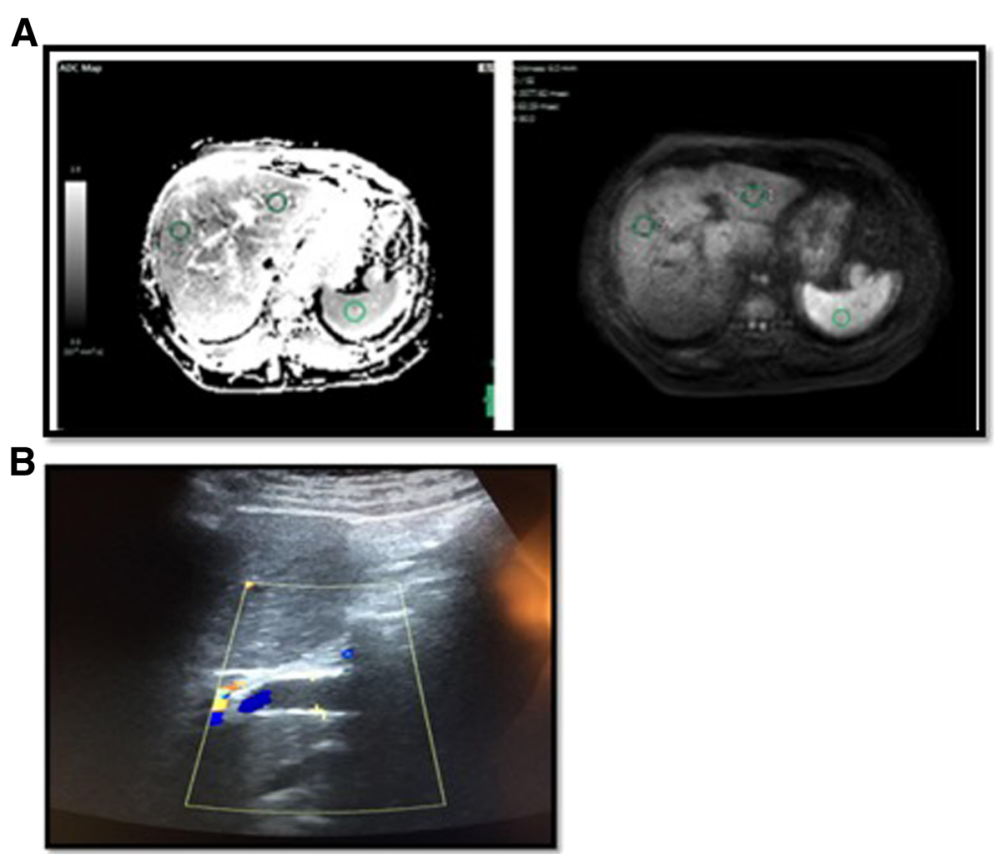

Fig. 6 a- 53 years old HCV positive male patient underwent Fibroscan with 6.3kPa which was classified as F1 stage. (F1=5-7 kPa), his Diffusion study: reveals mildly enlarged liver with no focal lesions. The spleen was of average size with no focal lesions. (a)- ADC map, and (b) - DWI at $b=$ 800- reveals liver mean $A D C=2.4 \times 10-3 \mathrm{~mm} 2 / \mathrm{s}$, and spleen mean $A D C=1.7 \times 10-3 \mathrm{~mm} 2 / \mathrm{s}$ so normalized $A D C=1.429(F 1 \mathrm{nADC}$ cut off $=1.44)$. b- His ultrasound study reveals mild hepatomegaly $(16.5 \mathrm{~cm})$ with no cirrhotic features or focal lesions. (c)-There was periportal fibrosis that can explain the low normalized ADC value as by further researches there might be subtypes in one stage 


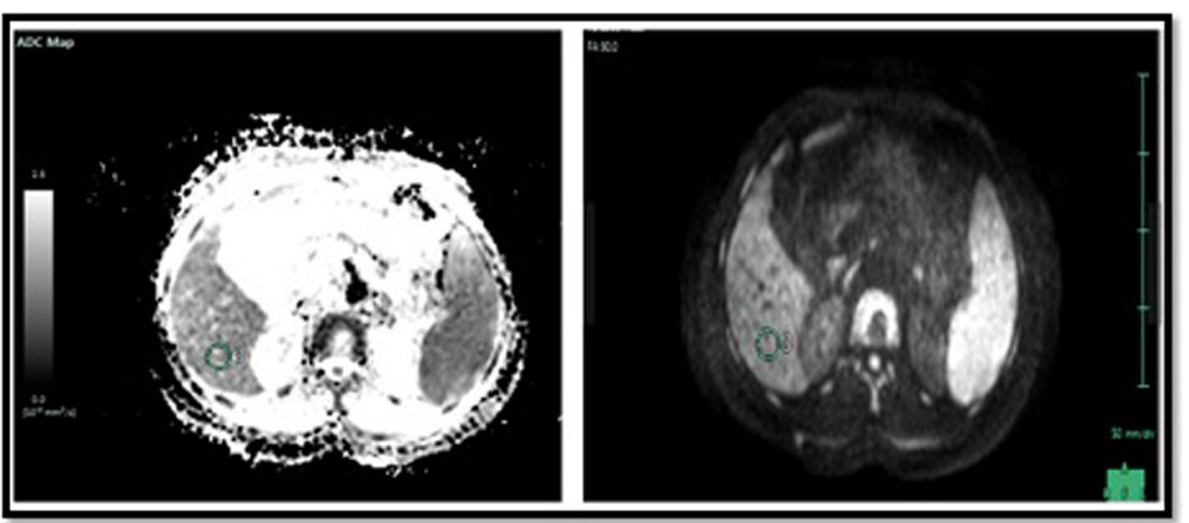

Fig. 745 years old HCV positive female patient, underwent Fibroscan with $14.5 \mathrm{kPa}$ which was classified as F4stage. (F4 > $12.5 \mathrm{kPa}$ ). His diffusion study reveals enlarged liver with cirrhotic features denoted by nodular hepatic surface, enlarged left lobe and deep fissures yet no focal lesions. The spleen was enlarged in size with no focal lesions. (a)-ADC map, and (b) - DWl at b $=800$ - reveals liver mean ADC $=1.6 \times 10-3 \mathrm{~mm} 2 / \mathrm{s}$, and spleen mean $A D C=1.7 \times 10-3 \mathrm{~mm} 2 / \mathrm{s}$ so normalized $A D C=0.94$. (F4 nADC cut off $=1.38)$

normalized liver ADC and ADC for all comparison's subsets except for diagnosis of cirrhosis (stage 4). nADC AUC was 0.878 for detection of $\geq$ F2 stages with sensitivity and specificity of $87 \%$ and $80 \%$ respectively while ADC value was 0.548 with sensitivity and specificity of $62 \%$ and $72 \%$ respectively ( $p=0.021$ ); nADC AUC for $\geq$ F3 stages was 0.891 with sensitivity and specificity of $88.7 \%$ and $80 \%$ respectively while ADC values were 0.603 with sensitivity and specificity of $72 \%$ and $72 \%$ respectively $(p=0.023)$, and F4 stage nADC AUC was 0.879 with sensitivity and specificity of $90 \%$ and $80 \%$ respectively, while ADC values were 0.648 with sensitivity and specificity of $80 \%$ and $72 \%$ respectively $(p=0.054)$. We acknowledge some limitations in current study as small number of subjects enrolled in this study and the lack of biopsy.

\section{Conclusion}

Using the spleen as a reference organ to calculate the normalized liver ADC increases the diagnostic performance of liver fibrosis than using ADC alone.

\section{Recommendation}

- We recommend using normalized ADC as a valid non-invasive option to diagnose and stage liver fibrosis in hepatitis $C$ patients. It can be used in obese, ascetic, or narrow intercostal space patients.

- Further studies are recommended with large participants' number and to be correlated with liver biopsy results.

- There is a potential of subgrouping each stage using color Doppler with the DWI.
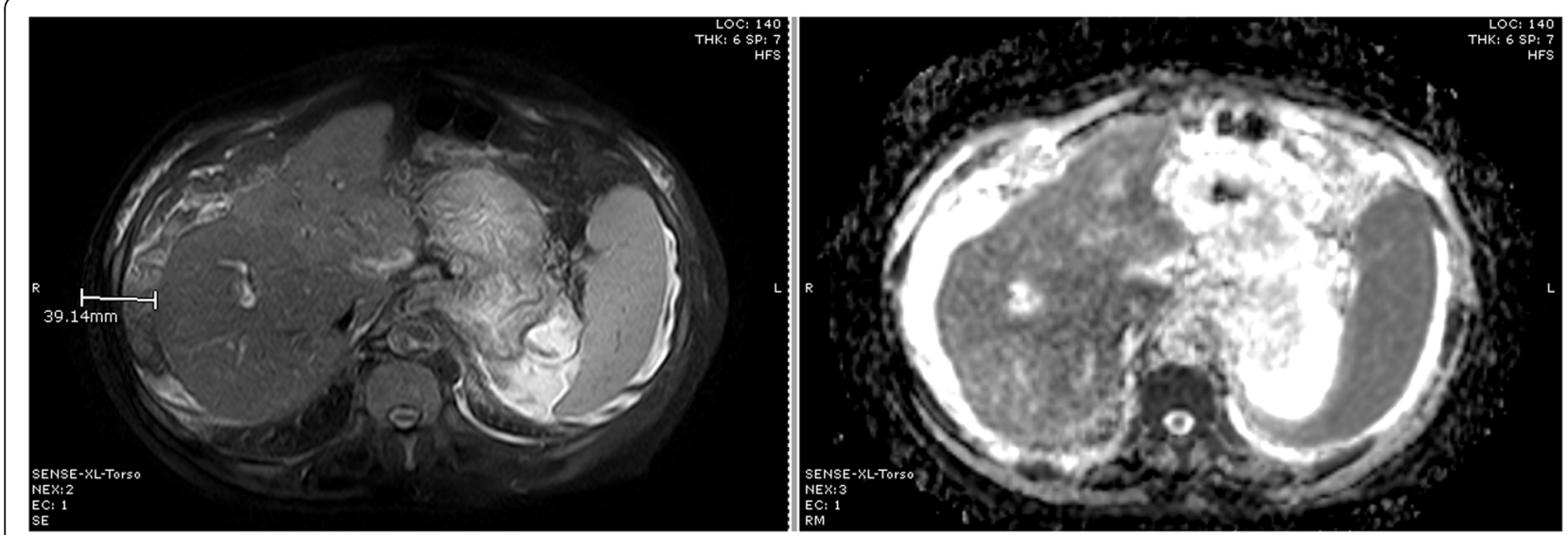

Fig. 8 Excluded case as there was moderate amount of ascites, it was classified as F4 by Fibroscan while mean liver ADC value $=1.5$ and mean spleen $\mathrm{ADC}=1.07$ and $\mathrm{nADC}=1.4$ 


\section{Abbreviations}

TE: Transient elastography; MR: Magnetic resonance; ADC: Apparent diffusion coefficient; nADC: Normalized apparent diffusion coefficient; PC: Personal computer (abbreviation added); DWl: Diffusion-weighted images;

HCV: Hepatitis C virus; ROI: Region of interest; EPI: Echo planner imaging; AUC: Area under the curve; ROC: Receiver operating characteristic curve

\section{Acknowledgements}

Our sincere thanks to all our professors and colleagues in the Radiology Department, Faculty of Medicine, Ain Shams University.

\section{Authors' contributions}

M. M collected, analyzed, and interpreted the patient data. O.K revised the results and was an editor in writing the manuscript. A. A revised the results and was a major contributor in writing the manuscript. M. A revised patients' data and was a major contributor in writing the manuscript. O. A referred the HCV patients to do Fibroscan examination. $\mathrm{H}$. A performed the Fibroscan examination. All authors read and approved the final manuscript.

\section{Funding}

No funds, sponsorship, or financial support to be disclosed.

\section{Availability of data and materials}

The datasets used and/or analyzed during the current study are available from the corresponding author on reasonable request.

\section{Ethics approval and consent to participate}

Research Ethics Committee at the Faculty of Medicine, Ain Shams University, has reviewed the study protocol from ethical point of view and approves it (FMASU MD445/2017)

All patients included in this research were fully conscious and older than 16 years old and gave written informed consent to participate in the study.

\section{Consent for publication}

All patients included in this research were fully conscious and older than 16 years old and gave written informed consent to publish the data contained within this study.

\section{Competing interests}

The authors declare that they have no competing interest.

\section{Author details}

${ }^{1}$ Radiology Department, Faculty of Medicine, Ain Shams University, Cairo, Egypt. ${ }^{2}$ Internal Medicine Department, Faculty of Medicine, Ain Shams University, Cairo, Egypt.

Received: 30 March 2020 Accepted: 27 May 2020

Published online: 26 June 2020

\section{References}

1. Feier D, Balassy C, Bastati N et al (2015) The diagnostic efficacy of quantitative liver MR imaging with diffusion-weighted, SWI, and hepatospecific contrast-enhanced sequences in staging liver fibrosis-a multiparametric approach. Eur Radiol 26(2):539-546

2. Castera $L$ (2012) Noninvasive methods to assess liver disease in patients with hepatitis B or C. Gastroenterology 142:1293-1302

3. Ghany MG, Nelson DR, Strader DB et al (2011) An update on treatment of genotype 1 chronic hepatitis C virus infection: 2011 practice guideline by the American association for the study of liver disease. Hepatology 54:14331444

4. El Refaei M (2015) Feasibility of different b-values of MRI diffusion in quantitative assessment of liver fibrosis. Journal of Cancer Prevention \& Current Research 2(2):00032

5. Kovač JD, Daković M, Stanisavljević D et al (2012) Diffusion-weighted MRI versus transient elastography in quantification of liver fibrosis in patients with chronic cholestatic liver diseases. Eur J Radiol 81(10):2500-2506

6. Taouli B, Chouli M, Martin AJ et al (2008) Chronic hepatitis: role of diffusionweighted imaging and diffusion tensor imaging for the diagnosis of liver fibrosis and inflammation. J Magn Reson Imaging 28:89-95

7. Foucher J, Castera L, Bernard PH et al (2006) Prevalence and factors associated with failure of liver stiffness measurement using FibroScan in a prospective study of 2114 examinations. Eur J Gastroenterol Hepatol 18 411-412

8. Bonekamp D, Bonekamp S, Ou HY et al (2014) Assessing liver fibrosis: comparison of arterial enhancement fraction and diffusion-weighted imaging. J Magn Reson Imaging 40:1137-1146

9. Richard K, Hersh C, Ely F et al (2010) Diagnosis of liver fibrosis and cirrhosis with diffusion weighted imaging: value of normalized apparent diffusion coefficient using the spleen as reference organ. AJR 195:671-676

10. Papanikolaou N, Gourtsoyianni S, Yarmenitis S et al (2010) Comparison between two-point and four-point methods for quantification of apparent diffusion coefficient of normal liver parenchyma and focal lesions: value of normalization with spleen. Eur J Radiol 73:305-309

11. Alavian SM, Miri SM, Keshvari M, Elizee PK, Behnava B, Tabatabaei SV, Lankarani KB (2009) Distribution of hepatitis C virus genotype in Iranian multiply transfused patients with thalassemia. Transfusion 49:2195-2199

12. Alavi S, Sadeghi E, Ashenagar A (2007) Efficacy and safety of combined oral iron chelation therapy with deferasirox and deferiprone in a patient with betathalassemia major and persistent iron overload. Blood Res 49:72-73

13. Strickland GT (2006) Liver disease in Egypt: hepatitis C superseded schistosomiasis as a result of iatrogenic and biological factors. Hepatology 43:915-922

14. Ziol M, Handra-Luca A, Kettaneh A et al (2005) Noninvasive assessment of liver fibrosis by measurement of stiffness in patients with chronic hepatitis C. Hepatology 41:48-54

15. Fraquelli M, Cassinerio E, Roghi A et al (2010) Transient elastography in the assessment of liver fibrosis in adult thalassemia patients. Am J Hematol 85: 564-568

16. Afdhal NH, Dieterich DT, Pockros PJ et al (2014) Epoetin alfa maintains ribavirin dose in HCV-infected patients: a prospective, double-blind, randomized controlled study. Gastroenterology. 126(5):1302-1311

17. Saito H, Tada S, Nakamoto $N$ et al (2004) Efficacy of non-invasive elastometry on staging of hepatic fibrosis. Hepatol Res 29(2):97-103

18. Tosun M, Inan N, Sarisoy HT et al (2013) Diagnostic performance of conventional diffusion weighted imaging and diffusion tensor imaging for the liver fibrosis and inflammation. Eur J Radiol 82:203-207. https://doi.org/ 10.1016/j.ejrad.2012.09.009

19. Taouli B, Koh DM (2010) Diffusion-weighted MR imaging of the liver. Radiology 254:47-46

20. Boulanger $Y$, Amara M, Lepanto L et al (2003) Diffusion-weighted MR imaging of the liver of hepatitis C patients NMR biomed, 16, pp. 132-136.

21. Marcellin P, Asselah T., Boyer N (2003): Fibrosis and disease progression in hepatitis C, Hepatology AASLD, Volume36, Issue5B, Pages s47-s56, //doi.org/ https://doi.org/10.1053/jhep.2002.36993

22. Bedossa $P$, Dargère $D$, Paradis V (2003) Sampling variability of liver fibrosis in chronic hepatitis C. Hepatology 38(6):1449-1457

23. Sandrasegaran K, Akisik F, Lin C et al (2009) Value of diffusionweighted MRI for assessing liver fibrosis and cirrhosis. AJR 193:1556-1560

24. Razek AK, Abdalla A, Omran E et al (2011) Diagnosis and quantification of hepatic fibrosis in children with diffusion weighted MR imaging. Eur J Radiol 78:129-134

25. Do RK, Chandanara H, Felker E et al (2010) Diagnosis of liver fibrosis and cirrhosis with diffusion-weighted imaging: value of normalized apparent diffusion coefficient using the spleen as reference organ. AJR 195:671-676

26. Taouli B, Koh DM (2010) Diffusion-weighted MR imaging of the liver. Radiology 254(1):47-66

27. Kandpal H, Sharma R, Madhusudhan KS et al (2009) Respiratorytriggered versus breath-hold diffusion- weighted MRI of liver lesions: comparison of image quality and apparent diffusion coefficient values. AJR 192:915-922

28. Shin S, Hwang $\mathrm{H}$ et al (2019) Liver fibrosis assessment with diffusionweighted imaging: value of liver apparent diffusion coefficient normalization using the spleen as a reference organ. Diagnostics 9(3):107

29. Kim T, Murakami T, Takahashi S et al (1999) Diffusion-weighted single-shot echoplanar MR imaging for liver disease. AJR 173:393-398

\section{Publisher's Note}

Springer Nature remains neutral with regard to jurisdictional claims in published maps and institutional affiliations. 